\title{
88. On the Respiratory Changes Induced by Sections of the Brainstem
}

\author{
Yoshiyuki NoguchI AND Sadao KAMII \\ Department of Orthopedic Surgery, Tokyo Dental College
}

The authors examined respiratory changes induced by sections of dog's brainstem. Respiratory tracing was carried out by means of tambour connected with the manchette around the animal's chest.

The results are as follows:

1. The authors affirm the existence of "pneumotaxic center", as they observed invariably sustained inspiratory apneusis by section of the cranial end of the pons caudal to inferior colliculi of the bilaterally vagotomixed and decerebrated animal.

2. Respiratory arrest occurs always immediately after transverse section of the medulla at the level of the obex, where as it is neither observed by cauterization of the floor of the fourth ventricle, nor by cauterization or section of the middle dorsal one-third or two-thirds of the medulla including the obex, nor by simple puncture into the obex until the base of the brain.

3. Respiratory arrest seemed to be produced by cut of the ventral part of the bilateral medial reticular formation at the level of the obex and by section of the ventrolateral part of the medulla at the level of the pyramidal decussation.

4. Longitudinal median section from the caudal end of the pons to the caudal end of the medulla elicits few respiratory changes. Therefore, fibers concerning respiration do not perhaps cross one another.

\section{Mesencephalic Micturition Inhibitory Area and the Pontine Micturition Facilitatory Area in the Cat. Their fiber Connections}

\author{
M. Kuru, H. Yamamoto, T. Iwanaga And Y. Koyama \\ The 2nd Surgical Division, Osaka University School of Medicine
}

After stimulating electrically the mesencephalic micturition inhibitory area (MMIA) described by Tang and Ruch and the pontine micturition facilitatory area (PMFA) described by Barrington and conforming the bladder responses, these areas were electrolytically destroyed and the fiber connections of these areas were demonstrated by Marchi method.

From MMIA degenerated fibers corresponding to tecto-pontine tract descend and terminating in the lateral portion of the ipsilateral pontine nucleus. The 